\title{
Artrite gotosa semelhante à artrite reumatoide: revisão de literatura
}

\author{
Gout arthitis similar to rheumathoid arthritis: literature review
}

Artritis gótica similar à la artritis reumatoid: revisión de la literatura

Larissa Marley Ribeiro de Sousa ${ }^{1 *}$, lane Brito Leal ${ }^{2}$, Sylvia Rannyelle Teixeira Lima ${ }^{3}$, Jaíse Mylena Passos e Silva ${ }^{1}$, Amélia Virgínia Ribeiro Araruna ${ }^{1}$, Larissa Petronio Sampaio ${ }^{1}$, Nathalia Cristine Nunes Menezes ${ }^{4}$, Maria Tayanne Parente Barbosa ${ }^{1}$, Ivan Júnior Miguel de Alencar ${ }^{5}$, Cecília Gomes dos Santos Alves ${ }^{3}$.

\section{RESUMO}

Objetivo: Descrever sobre artrite gotosa que assemelha à artrite reumatoide. Métodos: Trata-se de uma revisão bibliográfica narrativa. Foi realizada uma busca na base de dados em saúde PubMed. A estratégia de busca foi realizada com os seguintes descritores em inglês: "gout", "rheumatoid arthritis", "gout" AND "rheumatoid arthritis". Resultados: Ao utilizar o descritor "gout" AND "rheumatoid arthritis" foram encontrados 1.812 estudos científicos. Destes, foram selecionadas 329 produções que apresentavam o texto na íntegra, 188 relativos ao ano de publicação (entre 2009 e 2020) e 138 pertinente a espécie humana. Após a seleção por triagem pelos títulos e resumos restaram apenas 4 artigos científicos com os dois descritores. Ao abordar os descritores separadamente, a literatura é vasta e precisa. Todavia, quando abordados em conjunto a maioria dos estudos são relatos de caso esporádicos ao redor do mundo. Considerações finais: Assim, difundir o conhecimento sobre essa afecção e como manejá-la é mandatório para médicos, visto que esses pacientes podem permanecer por anos fazendo tratamentos incorretos que mascararam a real etiologia. Isso mostra a importância em voltar a atenção para o tema com a elaboração de mais estudos que possam estabelecer critérios diagnósticos diante de casos mais difíceis de chegar à etiologia.

Palavras-chave: Gota, Artrite reumatoide, Diagnóstico.

\begin{abstract}
Objective: To describe gout arthritis that resembles rheumatoid arthritis. Methods: This is a narrative bibliographic review. A search was performed in the PubMed health database. The search strategy was carried out with the following descriptors in English: "gout", "rheumatoid arthritis", "gout" AND "rheumatoid arthritis". Results/Discussion: Using the descriptor "gout" AND "rheumatoid arthritis", 1.812 scientific studies were found. Of these, 329 productions were selected that presented the full text, 188 for the year of publication (between 2009 and 2020) and 138 for the human species. After selection by sorting by titles and abstracts, only 4 scientific articles with two descriptors remained. When addressing the descriptors separately, the literature is vast and accurate. However, when approached together, most studies are sporadic case reports around the world. Conclusion: Therefore, spreading knowledge about this condition and how to manage it is mandatory for doctors, since these patients can remain for years doing incorrect treatments that have masked
\end{abstract}

\footnotetext{
${ }^{1}$ Faculdade de Medicina Estácio de Juazeiro do Norte (FMJ), Juazeiro do Norte - CE.

*E-mail: larimsousa10@gmail.com

2 Universidade Federal de Sergipe (UFS), Lagarto - SE.

${ }^{3}$ Universidade Federal do Cariri (UFCA), Barbalha - CE.

${ }^{4}$ Faculdade de Ciências Médicas de Campina Grande (UNIFACISA), Campina Grande - PB.

${ }^{5}$ Faculdade Federal do Piauí (UFPI), Picos - PI.
} 
the real etiology. This shows the importance of turning attention to the topic with the elaboration of more studies that can establish diagnostic criteria in the face of more difficult cases to reach the etiology.

Key words: Gout, Rheumatoid arthritis, Diagnosis.

\section{RESUMEN}

Objetivo: Describa sobre la artritis gotosa que se asemeja a la artritis reumatoid. Métodos: Esta es una revisión bibliográfica narrativa. Se realizó una búsqueda en la base de datos de salud PubMed. La estrategia de búsqueda se realizó con los siguientes descriptores en inglés: "gout", "rheumathoid arthritis", "gout" AND "rheumatoid arthritis". Resultados/Discusión: Usando el descriptor "gout" y " rheumathoid arthritis ", se encontraron 1.812 estudios científicos. De estos, se seleccionaron 329 producciones que presentaban el texto completo, 188 para el año de publicación (entre 2009 y 2020) y 138 para la especie humana. Después de la selección ordenando por títulos y resúmenes, solo quedaron 4 artículos científicos con dos descriptores. Al abordar los descriptores por separado, la literatura es amplia y precisa. Sin embargo, cuando se abordan juntos, la mayoría de los estudios son informes de casos esporádicos en todo el mundo. Conclusión: Por lo tanto, difundir el conocimiento sobre esta condición y cómo manejarla es obligatorio para los médicos, ya que estos pacientes pueden permanecer durante años haciendo tratamientos incorrectos que han enmascarado la verdadera etiología. Esto muestra la importancia de centrar la atención en el tema con la elaboración de más estudios que puedan establecer criterios de diagnóstico frente a casos más difíciles para llegar a la etiología.

Palabras clave: Gota, Artritis reumatoid, Diagnóstico.

\section{INTRODUÇÃO}

Artrite gotosa semelhante à artrite reumatoide (AR) é uma entidade clínica rara no espectro da medicina que pode gerar equívoco na etiologia das artrites. Define-se por início de sinais de inflamação articular em locais não-típicos da gota com crises recorrentes, prolongadas e poliarticulares que evolui para deformidades e podem mimetizar às da artrite reumatoide (CHAKRADEO K, et al., 2016; JEBAKUMAR AJ, et al., 2013; PEREIRA KD, et al., 2017; ZHAO G, et al., 2018).

Gota é considerada a forma mais comum de artrite em homens de meia idade e apresenta alta incidência e prevalência na reumatologia. Causada pela deposição de cristais de monourato de sódio na superfície articular quando os níveis de ácido úrico séricos estão elevados, entretanto, a hiperuricemia não é regra, visto que pode existir a deposição mesmo diante de níveis normais de urato. O foco é diminuir a alta morbidade gerada pelas ações irreversíveis ao atingir o estágio crônico (SARMENTO JF, et al., 2009; AZEVEDO VF, et al., 2017; PEREIRA KD, et al.,2017).

Nesse contexto, o padrão-ouro para diagnóstico é punção e análise do líquido sinovial que evidencia os cristais de monourato de sódio com birrefringência negativa. Contudo, estudos demonstram critérios clínicos que podem ser utilizados principalmente na atenção básica, onde o acesso à procedimentos invasivos é praticamente nulo.

Para AR, os critérios diagnósticos são bem estabelecidos pela Liga Europeia Contra Reumatismo (EULAR), ao utilizar basicamente de três vertentes: quadro clínico, exames laboratoriais e dosagem de anticorpos (KIM S, et al., 2014; STEINBERG J, et al., 2016; AZEVEDO VF, et al., 2017).

Com a finalidade de auxiliar no diagnóstico dessas duas entidades, os exames de imagem são elementares. A investigação tem início com radiografias das articulações acometidas, todavia não é um exame muito sensível.

Atualmente, a ultrassonografia (US) é aceita como exame de escolha para investigação inicial devido a possibilidade de visualizar alterações precoces nos tecidos periarticulares, de menor custo e sem radiação ionizante. Quando à US não é efetiva, pode utilizar a ressonância magnética para auxiliar. A tomografia 
computadorizada e a tomografia computadorizada de dupla energia são uteis por apresentar sensibilidade e especificidade elevadas e melhor avaliação de estruturas ósseas ( SARMENTO JF, et al., 2009; FERNANDES EDA, et al., 2017).

Em relação ao tratamento da artrite gotosa, enfatiza-se o entendimento do indivíduo sobre a cronicidade da doença, a importância de manter seguimento contínuo no tratamento, a modificação no estilo de vida e além da avaliação do risco cardiovascular (NUKI G, et al., 2016).

Nesse intuito, após o diagnóstico prossegue o tratamento medicamentoso de acordo com o estágio clínico da doença. Em situações de artrite gotosa aguda, a monoterapia com anti-inflamatórios não esteroidais (seletivos ou não) e colchicina é indicada.

Corticoides são indicados quando há falha ou contraindicação a primeira terapia. Já em artrites crônicas ou após a fase aguda, inicia o tratamento hipouricemiante que se fundamenta em modificação do estilo de vida e uso de agentes hipouricemiantes, alopurinol ou febuxostate na maioria das vezes (NUKI G, et al., 2016; AZEVEDO VF, et al., 2017).

O objetivo do estudo é realizar uma revisão sistemática através do que há de mais atual acerca da artrite gotosa que se assemelha à artrite reumatoide uma vez que este tema é pouco difundido o que pode gerar equívocos no diagnóstico e manejo.

\section{MÉTODOS}

A identificação e seleção dos estudos ocorreram entre os meses dezembro de 2019 e janeiro de 2020 , mediante a pergunta norteadora: "O que tem sido produzido na literatura sobre diagnóstico da gota em pacientes que iniciam quadro clínico clássico de artrite reumatoide?" Foi realizada uma busca na base de dados em saúde: PubMed.

A estratégia de busca foi realizada com os seguintes descritores em inglês e português, "gout", "rheumatoid arthritis", "gout" AND "rheumatoid arthritis". Foram considerados elegíveis estudos publicados em inglês, português e espanhol, nos últimos dez anos e disponível gratuitamente em formato eletrônico. Trabalhos duplicados na base de dados e que não tratem sobre Gota e Artrite Reumatoide em seus objetivos foram excluídos.

O processo de revisão consistiu em dois níveis de triagem, a revisão de título e resumo e a revisão do texto na íntegra. Para o primeiro, os títulos e resumos foram lidos e analisados para identificar artigos potencialmente elegíveis. Na segunda etapa, os artigos foram lidos na íntegra para determinar se atendem aos critérios de elegibilidade.

Para extração dos dados dos artigos selecionados foi elaborada uma ficha padrão com campos para coleta dos dados como, título, autores, ano de publicação, revista, tipo de estudo e local de realização. Todas as referências foram revisadas por completo extraindo fragmentos relevantes para o trabalho em forma de citação.

\section{RESULTADOS E DISCUSSÃO}

Inicialmente foram encontradas 18.268 produções científicas na PubMed com o descritor "gout". Destas, foram selecionadas 4.332 produções que apresentavam o texto na íntegra, disponível online. Apenas 2.879 atenderam aos critérios de elegibilidade relativo ao ano de publicação. Ao limitar a busca para espécie humana, restaram 1.827 artigos. Destes, somente 7 atenderam aos critérios de elegibilidade (Figura 1).

Ao utilizar o descritor "rheumatoid arthritis" foram encontrados 146.427 estudos científicos que, após aplicação dos critérios supracitados, foram selecionadas 2 produções. Com os descritores "gout" AND "rheumatoid arthritis" foram encontradas 1.812 produções. Destas, somente 6 atenderam aos critérios de elegibilidade (Figura 1). O Quadro 1 possibilita uma visão geral das 15 produções selecionadas: 


\section{Revista Eletrônica Acervo Saúde / Electronic Journal Collection Health ｜ ISSN 2178-2091}

Quadro 1 - Estudos incluídos na revisão e suas principais características.

\begin{tabular}{|c|c|c|c|c|c|}
\hline Título & Autores & $\begin{array}{c}\text { Ano de } \\
\text { publicação }\end{array}$ & Revista & Tipo de estudo & Local de realização \\
\hline $\begin{array}{l}\text { Artrite da gota tofácea crônica } \\
\text { mimetizando artrite reumatoide }\end{array}$ & Sarmento JF, et al. & 2009 & $\begin{array}{l}\text { Revista Brasileira de } \\
\text { Reumatologia }\end{array}$ & Relato de caso & Ambulatório \\
\hline $\begin{array}{l}\text { When Common Diagnosis Is Not } \\
\text { Common Enough }\end{array}$ & Senguttuvan NB, et al. & 2011 & $\begin{array}{l}\text { Internal Medicine } \\
\text { Journal }\end{array}$ & Relato de caso & Hospitalar \\
\hline Multiple arthritis: three in one & Henriques CC, et al. & 2012 & BMJ Case Reports & Relato de caso & Não especificado \\
\hline $\begin{array}{l}\text { Occurrence of gout in rheumatoid } \\
\text { arthritis: it does happen! A } \\
\text { population-based study }\end{array}$ & Jebakumar AJ, et al. & 2013 & $\begin{array}{l}\text { International Journal of } \\
\text { Clinical Rheumatology }\end{array}$ & Corte retrospectivo & $\begin{array}{l}\text { Registros médicos de } \\
\text { várias instituições de } \\
\text { saúde }\end{array}$ \\
\hline $\begin{array}{l}\text { Gout Initially Mimicking } \\
\text { Rheumatoid Arhritis and Later } \\
\text { Cervical Spine Involvement }\end{array}$ & Nunes EAS, et al. & 2014 & $\begin{array}{l}\text { Hindawi Publishing } \\
\text { Corporation }\end{array}$ & Relato de caso & Hospitalar \\
\hline $\begin{array}{l}\text { Global Metabolite Profiling of } \\
\text { Synovial Fluid for the Specific } \\
\text { Diagnosis of Rheumatoid Arthritis } \\
\text { from Other Inflammatory Arthritis }\end{array}$ & Kim S, et al. & 2014 & PLOS ONE & Experimental & Hospitalar \\
\hline $\begin{array}{c}\text { Adherence to the } 2012 \text { American } \\
\text { College of Rheumatology (ACR) } \\
\text { Guidelines for Management of } \\
\text { Gout: A Survey of Brazilian } \\
\text { Rheumatologists }\end{array}$ & Vargas-Santos $A B$, et al. & 2015 & PLOS ONE & Transversal & Não se aplica \\
\hline $\begin{array}{l}\text { Nodular rheumatoid arthritis } \\
\text { resembling gout }\end{array}$ & Chakradeo K, et al. & 2016 & BMJ Case Reports & Relato de caso & Ambulatório \\
\hline $\begin{array}{c}\text { Clinical Diagnosis of Gout Without } \\
\text { Joint Aspirate }\end{array}$ & Steinberg J, et al. & 2016 & $\begin{array}{l}\text { American Family } \\
\text { Physician }\end{array}$ & Experimental & Ambulatorial \\
\hline $\begin{array}{c}\text { Aspectos relevantes do } \\
\text { diagnóstico e seguimento por } \\
\text { imagem na gota }\end{array}$ & Fernandes EDA, et al. & 2017 & $\begin{array}{l}\text { Revista Brasileira de } \\
\text { Reumatologia }\end{array}$ & Revisão & Não se aplica \\
\hline
\end{tabular}


Revista Eletrônica Acervo Saúde / Electronic Journal Collection Health ｜ ISSN 2178-2091

\begin{tabular}{|c|c|c|c|c|c|}
\hline Título & Autores & $\begin{array}{c}\text { Ano de } \\
\text { publicação }\end{array}$ & Revista & Tipo de estudo & Local de realização \\
\hline $\begin{array}{l}\text { Revisão crítica do tratamento } \\
\text { medicamentoso da gota no Brasil }\end{array}$ & Azevedo VF, et al. & 2017 & $\begin{array}{l}\text { Revista Brasileira de } \\
\text { Reumatologia }\end{array}$ & Revisão & Não se aplica \\
\hline $\begin{array}{c}\text { Gota Tofácea Crônica Erosiva } \\
\text { Semelhante À Artrite Reumatoide: } \\
\text { Relato De Caso }\end{array}$ & Pereira KD, et al. & 2017 & $\begin{array}{l}\text { Revista Brasileira de } \\
\text { Reumatologia }\end{array}$ & Relato de caso & Ambulatorial \\
\hline $\begin{array}{l}\text { Current management of gout: } \\
\text { practical } \\
\text { messages from } 2016 \text { EULAR } \\
\text { guidelines }\end{array}$ & Nuki G, et al. & 2016 & $\begin{array}{l}\text { Polish Achives of } \\
\text { Internal Medicine }\end{array}$ & Revisão & Não se aplica \\
\hline $\begin{array}{l}\text { Recognition of gout in rheumatoid } \\
\text { arthritis }\end{array}$ & Zhao G, et al. & 2018 & Wolters Kluwer Health & Relato de caso & Hospitalar \\
\hline $\begin{array}{c}\text { Does early seronegative arthritis } \\
\text { develop into rheumatoid arthritis? } \\
\text { A 10-year observational study }\end{array}$ & Paalanen $\mathrm{K}$, et al. & 2019 & $\begin{array}{l}\text { Clinical and } \\
\text { Experimental } \\
\text { Rheumatology }\end{array}$ & Observacional & Hospitalar \\
\hline
\end{tabular}

Fonte: SOUSA LMR, et al., 2020. 
Figura 1 - Fluxograma da seleção dos estudos.

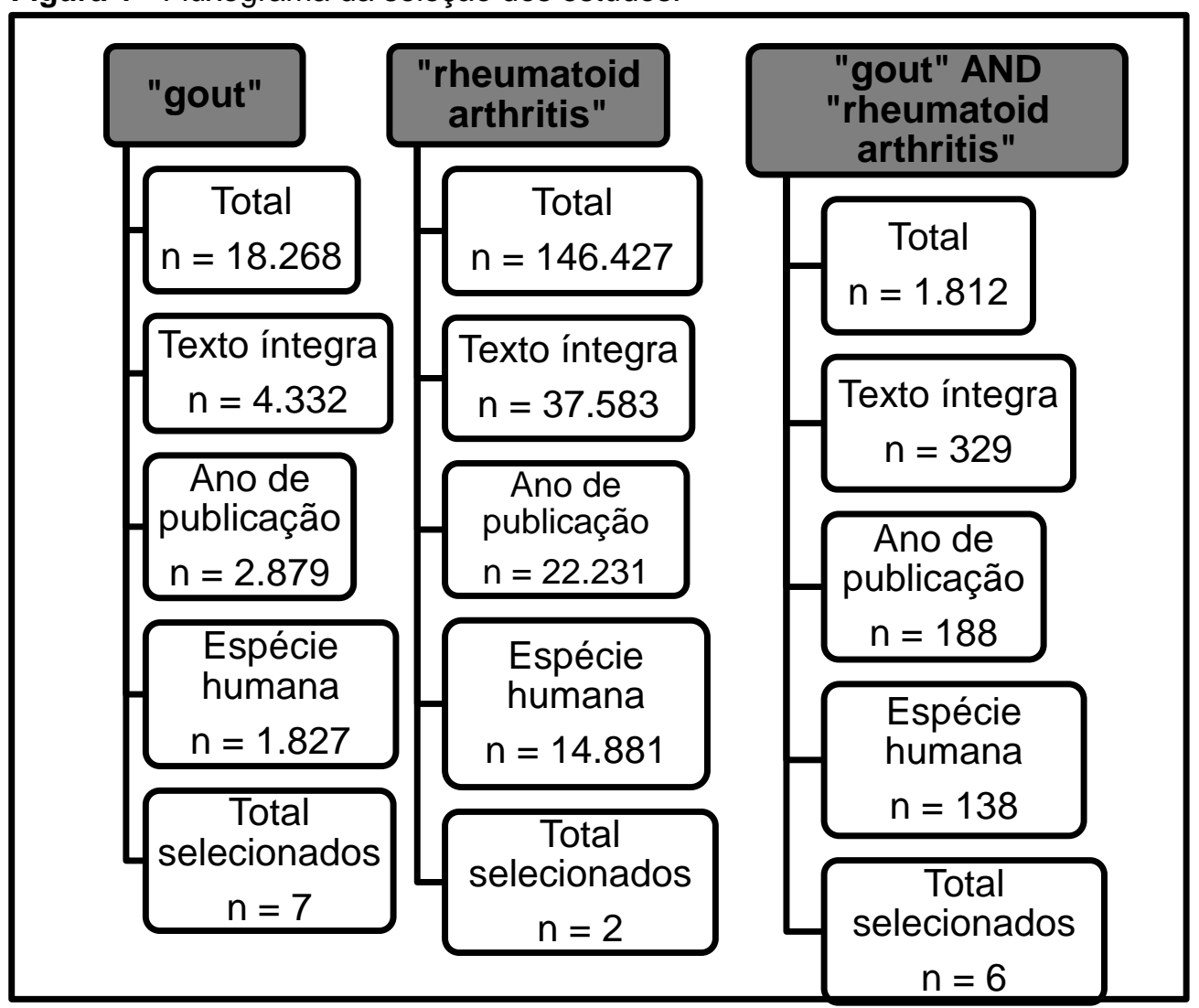

Fonte: SOUSA LMR, et al., 2020.

Diante do encontrado, evidenciou-se que a literatura ainda é escassa relacionada ao tema exposto. Segundo os autores Sarmento JF, et al. (2009) Jebakumar AJ, et al. (2013), Nunes EAS, et al. (2014) Chakradeo K, et al. (2016) Henriques, et al. (2012) e Zhao G, et al. (2018) isso se deve ao fato de que a artrite gotosa ao mimetizar artrite reumatoide (AR) ou a presença das afecções concomitantemente apresentam baixa incidência populacional, consideradas raras. Acredita-se que os cristais de urato podem bloquear a ativação de células $B$ e T além de propriedades antifagocitose nos macrófagos que colabora na redução da incidência concomitante de gota e AR no mesmo paciente (ZHAO G, et al., 2018). Pode ser difícil distinguir clinicamente as duas patologias principalmente quando a artrite gotosa é poliarticular e envolve mãos (JEBAKUMAR AJ, et al., 2013).

Dos estudos selecionados, apenas seis relatos de caso retrataram a presença de gota semelhante a artrite reumatoide ou ambas em um mesmo paciente. Sarmento JF, et al., (2009) aborda o caso de poliartrite simétrica em metacarpofalangianas e interfalangianas proximais, com rigidez matinal de mais de 1 hora e fator reumatoide positivo, com principal diagnóstico diferencial a artrite gotosa pela hiperuricemia apresentada pelo paciente.

A análise do líquido sinovial pôde estabelecer o diagnostico etiológico com posterior melhora clínica após tratamento hipouricemico. Chakradeo K, et al. (2016) demonstraram um paciente com poliartrite de mãos com anticorpo pepitídio anticitrulinado (anti-CCP) e fator reumatoide (FR) positivos que estabeleceu e tratou como AR. Todavia, diante do aparecimento de nódulos subcutâneos semelhantes a tofos gotosos, fora realizada histopatologia dos nódulos e definida área central de necrose com granulação, além de birrefringência positiva, que estabeleceu quadro de AR semelhante a gota.

Nunes EAS, et al. (2014) aborda um caso com quadro clínico clássico de AR, com deformidades em mãos em "pescoço de cisne" em punhos "dorso de camelo". Diante do aparecimento de nódulos subcutâneos e evidenciada hiperuricemia foi feito diagnóstico diferencial com gota tofácea crônica e confirmado após FR e anti-CCP negativos. O paciente evoluiu com acometimento cervical e paralisia braquial à esquerda sendo 
submetido a neurocirurgia e tratamento hipouricemiante concomitantemente. De acordo com a literatura, raros casos de gota que envolve esqueleto axial foram relatados, principalmente os associados à compressão medular. Ademais, alguns casos de acometimento cervical foram descritos mesmo sem história previa de artrite. Devido à raridade desses casos, é necessário excluir etiologias como espondiloartrites. Para isto é de fundamental importância exames de imagem.

Um caso atípico descreve a presença de três artrites de etiologias diferentes em único paciente (AR, artrite psoriática e artrite gotosa). De acordo com os critérios da EULAR, do ACR e dos Critérios Classificatórios para Artrite Psoriática (CASPAR) foi possível inclui-lo inicialmente em duas entidades autoimunes. A princípio, artrite psoriásica com posterior diagnóstico de artrite gotosa confirmada através da análise do líquido sinovial. Apresentou poliartrite assimétrica bilateral em metacarpofalangianas e deformidades articulares, além de tofos no lóbulo da orelha, mãos e pés.

A positividade do FR e do anti-CCP em conjunto com exames de imagem sugestivos e biópsia dos nódulos, confirmou AR sobreposta. O mais significativo do caso foi o diagnóstico etiológico precoce das três afecções em que o tratamento instituído para cada uma delas não exacerbasse as outras. O uso de anticorpos contra o fator de necrose tumoral para tratamento de AR paradoxalmente leva a uma piora da artrite psoriásica. (HENRIQUES CC, et al., 2012).

Senguttuvan NB, et al. (2011) elencam um caso de artrite em todas as articulações dos membros inferiores e superiores simetricamente com diagnostico de AR. Ao tratamento a melhora foi parcial. Contudo, ao exame clínico apresentou deposição de tofos no lóbulo da orelha, pés e dedos. Ao exame radiográfico, pode-se notar o Sinal de Martel (artrite erosiva com lesões de bordos salientes). Ademais, foi demonstrada hiperuricemia que evoluiu com insuficiência renal aguda e diagnóstico de gota tofácea crônica deformante.

Em crises de artrite prolongadas e poliarticulares, Pereira KD, et al. (2017) afirmam que o diagnóstico se assemelha à AR, diferentemente da gota que se caracteriza pela monoartrite aguda e intermitente. Deste modo, relatam um caso de artrite de primeira metatarsofalangiana e joelhos com provas de atividade inflamatória elevadas e com FR e anti-CCP negativos.

Todavia, o longo período sem tratamento do paciente, evoluiu com poliartrite simétrica de interfalangianas proximais, metacarpofalangianas, punhos, cotovelos, joelhos, tornozelos e metatarsofalangianas com rigidez matinal intensa e prolongada além de deformidades em mão e tofos em cotovelos. À radiografia foi evidenciada lesões em "saca-bocado" que sugere fortemente o diagnóstico de gota tofácea crônica.

Zhao G, et al. (2018) descreve um caso de poliartrite com rigidez matinal importante e diminuição do espaço articular à radiografia. Evidenciados FR e anti-CCP positivos, ácido úrico sérico dentro da normalidade e fator antinuclear com título de 1:1000. Isto o incluiu de acordo com o critério estabelecido pela EULAR para AR. Após 13 anos de tratamento contínuo, evoluiu com artrite em joelhos que após US evidenciou o "sinal de via dupla", análise do líquido sinovial com cristais com birrefringência negativa e hiperuricemia. Por fim, foi estabelecido o diagnóstico de AR complicada com artrite gotosa.

Um estudo de coorte retrospectivo populacional pode demonstrar a ocorrência de gota em pacientes com diagnóstico prévio de AR. Foram selecionados 813 pacientes entre 1 de janeiro de 1980 e 31 de dezembro de 2007, destes apenas 22 tiveram o diagnóstico de gota concomitante com artrite reumatoide. A prevalência de gota em pacientes com AR foi significativamente menor em relação à população geral.

Dentre os motivos elencados, incluem: a maior excreção renal de ácido úrico em mulheres (maior prevalência de AR) causada pelo estrógeno e progesterona; o uso de corticoides na AR que podem camuflar o quadro clinico; os cristais de urato que podem bloquear a ativação de células B e T; além da interleucina 6 envolvida na fisiopatologia da AR que tem propriedades uricosúricas. Foram identificados como principais fatores de risco para gota em pacientes com AR aqueles do sexo masculino, idade mais avançada $e$ obesidade, os quais são também para a população geral (JEBAKUMAR AJ, et al., 2013).

Em estudo observacional que abordou a evolução de artrite inicialmente soronegativa para AR em 10 anos, foi constatado que há grande heterogeneicidade em pacientes inicialmente diagnosticados com AR. Foram avaliados os principais grupos de artrites dentro da reumatologia: espondiloartrites, polimialgia 
reumática, vasculites, artrites causadas por cristais e osteoartrite. Ao abordar gota, pseudogota e doença por deposição de pirofosfato de cálcio observou-se que independente do quadro clínico ser típico ou não, ao constatar cristais na análise do líquido sinovial, o diagnóstico era estabelecido, caso contrário, era reclassificado (PAALANEN K, et al., 2019).

Em relação ao diagnóstico, o padrão-ouro para gota é a identificação de cristais com birrefringência negativa na microscopia de luz polarizada em material aspirado em artrocentese da articulação acometida, de preferência durante a crise aguda (ZHANG W, et al., 2006).

Não obstante, Steinberg J, et al. (2016) demonstraram por regressão logística os sete melhores preditores de gota, voltados principalmente para médicos da atenção primária, visto que a maioria dos indivíduos não chegam à atenção secundária com reumatologista. São eles: início agudo de aproximadamente um dia (0,5 pontos); eritema articular (1 ponto); hipertensão arterial sistêmica ou doença cardiovascular, angina, infarto agudo do miocárdio, acidente vascular cerebral, ataque isquêmico transitório e doença vascular periférica, (1,5 pontos); sexo masculino (2 pontos); ataque anterior de artrite ou artralgia (2 pontos); primeira metatarsofalangeana envolvida (2,5 pontos) e ácido úrico sérico acima de 5,8 mg/dl (3,5 pontos).

A soma dos escores classifica o indivíduo: acima de 8 pontos (alto risco) é dado diagnóstico de gota. Entre 4,5 e 7,5, risco intermediário, a conduta é realizar aspiração do líquido sinovial e considerar o encaminhamento ao reumatologista. Caso não seja opção, é recomendado a reavaliação entre 3 e 6 meses. Abaixo de 4 pontos (baixo risco) o diagnóstico é descartado.

Ao discorrer sobre AR, Kim S, et al. (2014) examinaram e abordaram as alterações do metabolismo do líquido sinovial nas articulações acometidas em pacientes ainda sem diagnóstico definitivo e com a finalidade de encontrar potenciais marcadores para diagnóstico na artrocentese, elaboraram o perfil metabólico global para o diagnóstico específico de AR ao utilizar cromatografia gasosa e espectrometria de massa de campo de voo.

Nas análises estatísticas dos metabólitos dos 38 pacientes selecionados (com diagnóstico prévio de AR, espondilite anquilosante, doença de Behçet e gota) encontrou-se uma sensibilidade de $92,3 \%$ e especificidade de $68 \%$ para 20 metabólitos, são eles: succinato, octadecanol, asparagina, tereftalato, salicilalideido, glutamina, citrulina, tirosina, uracil, lisina, ribitol, triptofano, xilose, ribose, ácido isopalmítico, glicerol, ácido mirístico, ácido palmitoleico, hidroxilamina e etanolamina.

Diante das manifestações atípicas, os exames de imagem têm papel fundamental. Em artigo de revisão publicado por Fernandes EDA, et al. (2017) foram analisados os métodos de imagem mais utilizados no Brasil para diagnóstico de gota além de demonstrar os mais indicados na prática clínica. Dentre eles, a radiografia é o mais utilizado pelo baixo custo e facilidade, porém tem pouca utilidade ao ser pouco sensível nas fases mais precoces da doença enquanto na fase mais tardia pode aparecer a reação de Martel (borda óssea separada e inclinada sobre o tofo) além de preservação do espaço articular, erosões corticais e lesões líticas. Ultrassonografia foi eleito o método de imagem mais indicado por apresentar bons resultados, não ser invasivo e difusamente disponível.

Alguns sinais característicos podem auxiliar no diagnostico como o "sinal do duplo contorno" (camada hiperecoica linear e irregular sobre a cartilagem hialina sem sombra acústica posterior) e focos puntiformes hiperecoicos que associados podem chegar a $100 \%$ de especificidade.

Além disso, o estudo ampliado com Doppler pode demonstrar aumento de fluxo na fase aguda da doença e sem fluxo no período intercrítico. Dimensões do tofo e avaliação de resposta ao tratamento também podem ser evidenciadas por US.

Em relação à tomografia computadorizada, sua importância se dá quando há acometimento de estruturas mais profundas, ao passo que na avaliação de estruturas superficiais não é preconizada como método de escolha. Já a tomografia computadorizada de dupla energia, ao demonstrar a composição química dos tecidos e os diferenciar, é passível de identificar os cristais de monourato de sódio, contudo a utilização é restrita a ensaios clínicos de novos agentes medicamentosos. 
Quanto a ressonância magnética, fornece informações sobre a morfologia dos tofos e pode detectar alterações precoces de erosões articulares não vistas nas radiografias, porém são inespecíficas. Portanto, sem uso rotineiro na prática clínica para diagnóstico de gota (FERNANDES EDA, et al., 2017).

Azevedo VF, et al. (2017) em artigo de revisão sobre tratamento da gota, enfatizam as possibilidades estabelecidas e as novas viabilidades para isto. Para crises de mono ou oligoartrite agudas é indicada monoterapia com duas principais drogas atualmente: anti-inflamatórios não-esteroidais (AINEs) ou esteroidais e colchicina.

Em relação aos AINEs, não existem diferenças marcantes de acordo com o perfil de melhor escolha para esses pacientes. Tanto os seletivos (inibidores da cicloxigenase-1) quanto os não-seletivos são semelhantes em sua eficácia. Todavia são distinguíveis no perfil de efeitos colaterais e segurança cardiovascular. Uma opção viável, seria a adição de Inibidores de Bomba de Prótons (IBPs) ao tratamento nos pacientes passíveis de uso de AINEs não-seletivos, além de se tornar um tratamento mais barato, visto o alto custo dos inibidores seletivos da COX-1 (AZEVEDO VF, et al., 2017).

Em relação à colchicina, sabe-se que sua atuação ao diminuir a atividade dos neutrófilos e inibir a formação do inflamassomo NLRP3, é útil no tratamento da artrite gotosa aguda. É importante antes de iniciar o tratamento, obter funções renal e hepática. O ACR acrescenta que os melhores resultados em relação a droga se estabelecem com administração 24 horas do início dos sintomas e não ultrapassar 36 horas.

O uso dos corticosteroides fica restrito quando há falha ou contraindicação ao tratamento anterior. O uso do corticóide intra-articular após a aspiração da articulação acometida é considerado padrão ao reduzir mais rapidamente os sintomas e ter menor perfil dos efeitos colaterais (AZEVEDO VF, et al., 2017).

Ademais, outra terapia alvo é a inibição da interleucina-1 com medicamentos biológicos que fica restrita a pacientes que se encontrarem em falha terapêutica ou quando não for possível utilizar os medicamentos supracitados. A desvantagem desse tratamento se resume ao seu alto custo e dificuldade de obtenção no Sistema Único de Saúde (SUS) no Brasil (AZEVEDO VF, et al., 2017).

A terapia de longo prazo se baseia no tratamento hipouricemiante com objetivo de manter a uricemia abaixo de $6 \mathrm{mg} / \mathrm{dl}$. Para isto, a recomendação é a interação entre dieta hipouricemiante e tratamento de comorbidades. Com diagnóstico bem estabelecido, é mandatória a utilização dos inibidores da xantina oxidase (alopurinol e febuxostate).

O alopurinol é considerado de escolha no Brasil pela facilidade de acesso, efetividade, comodidade posológica e segurança. Contudo, possui limitações em indivíduos com insuficiência renal ou em uso de diurético. O febuxostate, fica reservado aos com doença renal crônica, porém é contraindicado em pacientes com insuficiência cardíaca. No entanto, não há previsão de entrada desta droga no país (AZEVEDO VF, et al., 2017).

Outra opção são os agentes uricosúricos, porém ficam reservados como segunda linha por aumentar a incidência de cálculos renais. A importância do tratamento hipouricemiante não se resume a profilaxia de novos eventos agudos de artrites, mas à diminuição no risco cardiovascular comprovado na literatura (AZEVEDO VF, et al., 2017; NUKI G, et al., 2016).

Em um estudo sobre a adesão dos reumatologistas brasileiros, em relação as recomendações do ACR, Vargas-Santos AB, et al., (2015) selecionaram 304 profissionais que foram submetidos a um questionário online durante período de 3 meses. As ações abordadas foram: terapia para crise aguda, tratamento hipouricemiante e profilaxia. Além disso, foram levantados dados demográficos, experiência na área de reumatologia e número de pacientes mensais com diagnóstico de gota. Observou-se discordância entre o resultado das amostras e as recomendações pelo ACR nos três âmbitos de tratamento.

A primeira delas corresponde a utilização da colchicina acima de 36 horas da crise aguda, além de doses acima de $2 \mathrm{mg}$ ao dia (maior chance de toxicidade). Além disso, a duração da profilaxia (alguns indefinidamente outros com menos de um mês) demonstrou que o tempo estabelecido era muito longo ou muito curto, sem benefício ao indivíduo em ambos os casos. 
Outra refere-se à cessação dos agentes hipouricemiantes durante ataque agudo de gota em pacientes com tratamento profilático. Segundo as recomendações da ACR, confirmado através desse estudo, a maioria dos reumatologistas interrompem o tratamento em vigência de crise, porém isto prolonga e torna mais difícil controlar o quadro. Além disso, doses iniciais de $300 \mathrm{mg} /$ dia foram relatadas por $42,7 \%$ dos reumatologistas sendo inconsistente com o recomendado. As discordâncias encontradas dizem respeito ao tratamento de crise de artrite gotosa aguda, prescrição de profilaxia hipouricemiante, monitorização e alvo de uricemia (VARGAS-SANTOS AB, et al., 2015).

Para Vargas-Santos AB, et al. (2015) as recentes mudanças no manejo da doença podem não ter sido difundidas numa proporção significativa. Os principais fatores apontados no estudo para isto foram: reumatologistas mais velhos ou com pouco tempo de prática e os que trabalham exclusivamente na área privada.

\section{CONSIDERAÇÕES FINAIS}

Gota e artrite reumatoide são afecções prevalentes dentro do espectro clínico da reumatologia, porém quando há presença de uma, geralmente a outra não se desenvolve. A existência de ambas no mesmo paciente apesar de rara, é importante ser relatada com a finalidade de estabelecer o diagnóstico precoce e evitar sua morbidade. Diante disso, a importância do tema se baseia no diagnóstico etiológico de artrites que se desenvolvem de formas atípicas. Quando abordadas separadamente, a literatura é vasta e precisa para o manejo de ambas. Todavia, quando abordadas em conjunto a maioria dos estudos são relatos de caso esporádicos ao redor do mundo. Isso mostra a importância em voltar a atenção para o tema com a elaboração de mais estudos que possam estabelecer critérios diagnósticos diante de casos mais difíceis de chegar à etiologia.

\section{REFERÊNCIAS}

1. AZEVEDO VF, et al. Revisão crítica do tratamento medicamentoso da gota no Brasil. Revista Brasileira de Reumatologia, 57(4), 346-355, 2017.

2. CHAKRADEO K, et al. Nodular rheumatoid arthritis resembling gout. BMJ Case Reports, 2016, 1-2, 2016.

3. FERNANDES EDA, et al. Relevant aspects of imaging in the diagnosis and management of gout. Revista Brasileira de Reumatologia, 57(1), 64-72, 2017.

4. HENRIQUES CC, et al. Multiple arthritis : three in one. 2011-2013, 2012.

5. JEBAKUMAR, AJ, et al. Occurrence of gout in rheumatoid arthritis: It does happen! A population-based study. International Journal of Clinical Rheumatology, 8(4), 433-437, 2013.

6. KIM S, et al. Global Metabolite Profiling of Synovial Fluid for the Specific Diagnosis of Rheumatoid Arthritis from Other Inflammatory Arthritis. 9(6), 1-9, 2014.

7. NUKI G, et al. Current management of gout : practical messages from 2016 EULAR guidelines. 267-277, 2016.

8. PAALANEN K, et al. Does early seronegative arthritis develop into rheumatoid arthritis? A 10-year observational study. Clinical and Experimental Rheumatology, 37(1), 37-43, 2019.

9. PEREIRA KD, et al. Gota Tofácea Crônica Erosiva Semelhante À Artrite Reumatoide: Relato De Caso. Revista Brasileira de Reumatologia, v. 57, n. S 1, p. S126-S127, 2017.

10. SARMENTO JF, et al. Artrite da gota tofácea crônica mimetizando artrite reumatoide. Revista Brasileira de Reumatologia, 49(6), 741-746, 2009.

11. SENGUTTUVAN NB, et al. When common diagnosis is not common enough. Internal Medicine, v. 50, n. 24, p. 30453046, 2011.

12. STEINBERG J, et al. Point-of-Care Guides Clinical Diagnosis of Gout Without Joint Aspirate,2016.

13. VARGAS-SANTOS AB , et al. Adherence to the 2012 American college of rheumatology (ACR) guidelines for management of gout: A survey of Brazilian rheumatologists. PLoS ONE, 10(8), 1-17, 2015.

14. ZHANG W, et al. EULAR evidence based recommendations for gout. Part II: Management. Report of a task force of the EULAR Standing Committee for International Clinical Studies Including Therapeutics (ESCISIT). Annals of the Rheumatic Diseases, 65(10), 1312-1324, 2006.

15. ZHAO G, et al. Recognition of gout in rheumatoid arthritis A case report. Medicine (United States), 97(50), 4-7, 2018. 